\title{
CINEMA-CLASS DIGITAL CONTENT DISTRIBUTION VIA OPTICAL NETWORKS Invited paper
}

Tetsuro Fujii, Kazuhiro Shirakawa, Mitsuru Nomura, and Takahiro Yamaguchi NTT Network Innovation Laboratories 1-1 Hikarinooka Yokosuka-shi,Knanagawa 239-0847 Japan

fujii.tetsuro@lab.ntt.co.jp

Abstract: $\quad$ To transmit and display high quality movies via optical networks, a new Super High Definition (SHD) digital cinema distribution system with the resolution of 8-million pixel is developed. Its image quality is four times of HDTV in resolution, and enables us to replace conventional $35 \mathrm{~mm}$ films. This system is based on JPEG 2000 coding technology and transmits high quality digital cinema over high-speed IP networks. All digital cinema data are continuously transmitted at up to $500 \mathrm{Mbps}$. This system opens the door to the next generation of cinema-class digital content distribution over optical networks.

\section{INTRODUCTION}

The growth of broadband networks has stimulated the development of applications that use high quality image communications. To satisfy professional users in industry, i.e. printing, medicine, and image archiving, a precision color imaging system is required to achieve the digital images of excellent quality beyond HDTV. An image category, called Super High Definition (SHD) images $[1,2]$ is defined to have a resolution of at least 2000 pixels vertically with 24 -bit color separation. The SHD images surpass the quality of $35-\mathrm{mm}$ films in terms of spatial resolution. In our first study on the SHD images and their applications, we developed a high quality still image system with 28.3 inches LCD display of $2560 \times 2048$ pixel resolution. This image system features GbE as high speed 
network interface and can transmit 2000 scanning line class still images within one second.

At the same time, we have developed a new platform for high quality digital cinema with 8 million pixels, called SHD digital cinema. This SHD digital cinema scales the heights now occupied by $35 \mathrm{~mm}$ film. It offers large venue support, large screen projection to fully realize the promise of digital cinema, scalable integrated media production, and film-less cinema distribution via broadband networks. The new SHD digital cinema format is defined as 2000 or more scan lines, progressively scanned, running at 24 frames per second. To evaluate this new format, we have developed real-time DECODER and a projector capable of handling SHD digital cinema with an effective resolution of $3840 \times 2048$ pixels (square sample). SHD digital cinema contains roughly four times the picture information of HDTV 1080p/24. A comparison is made in Figure 1. SHD digital cinema features RGB color encoding and 30 bits per pixel, for a much more filmlike visual richness. Motion picture people in Hollywood count up the cinema resolution from the point of horizontal pixel. Therefore, they call our system as "4K Digital Cinema".

To transmit the movie contents using optical networks, an exceptionally high performance decoder and an imaging system are required to process the movies in real-time. This is because the total bit rate of an SHD digital cinema can equal 5.6 Gbps (3840 x 2048 pixels, 24 fps and 30-bit color), and the movie should be compressed to 10:1 - 20:1 in order to transmit them by wide-area IP networks. Eventually a special combination of a real-time DECODER and a projection device is required to show SHD digital cinema. In this paper, we introduce an SHD digital cinema distribution system.

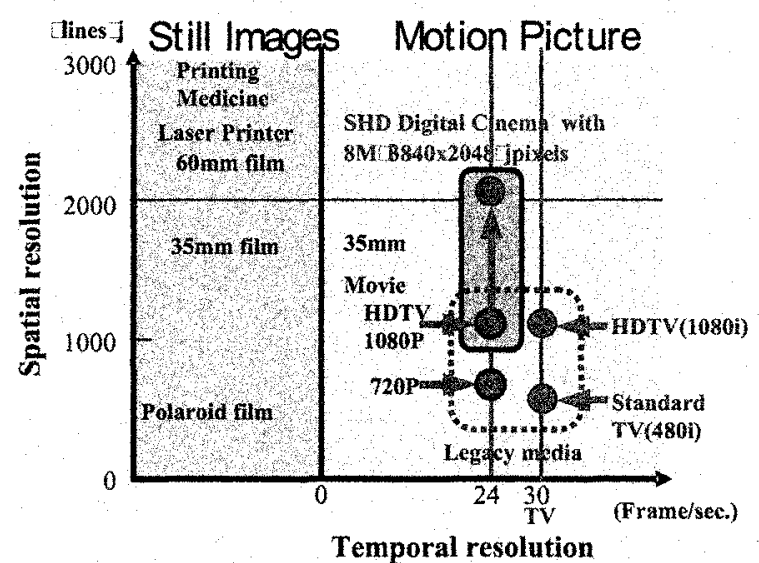

Figure 1. Resolution and frame rate of SHD images 


\section{SHD DIGITAL CINEMA SYSTEM}

We have developed a prototype digital cinema system that can store, transmit and display SHD digital cinema of 8 million (3840x2048) pixel resolution using JPEG2000[3] coding algorithm. The SHD digital cinema distribution system is shown in figure 2 and 3. This is the third generation of our SHD digital cinema distribution system[4,5,6]. The transmission from the server to the real-time DECODER is done over GbE (Gigabit Ethernet). It consists of three main devices, a video server, a real-time DECODER, and a LCD projector. We assume that the movie data have been compressed and stored in advance. The real-time DECODER decompresses the video streams transmitted from the server using parallel JPEG2000 processors, and outputs the digital video data to an LCD projector with $3840 \times 2048$ pixel resolution and RGB 30 bits with 24 fps.

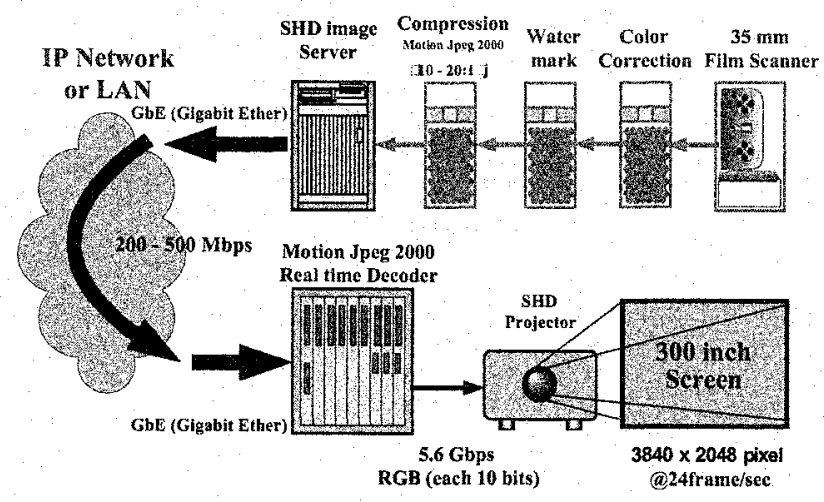

Figure 2. System conciguration of prototype SHD digital cinema distribution system

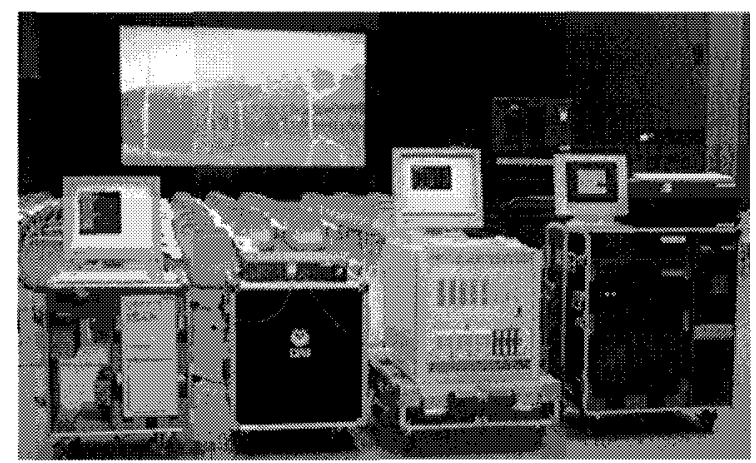

Figure 3. Photograph of SHD digital cinema distribution system 


\subsection{Image Coding Algorithm:}

The SHD digital cinema system uses the JPEG2000 algorithm to construct a real-time DECODER. From the point of communication traffic and storage cost, inter-frame coding such as MPEG-2 is better at compressing movie data. However, we selected JPEG2000 for the following five reasons. (1) There is no international standard to compress video for RGB 30 bits or more. (2) Other sets of compressed data with lower resolution can be generated easily by using the facility for embedded scheme of layered coding algorithm. (3) Intra-frame coding schemes remain important because of their support of video editing. (4) It is much easier to implement a parallel processing decoder by using compact JPEG2000 CODEC chips. (5) The decoder is robust against data error. Error recovery is achieved simply by discarding the corrupted image frame. The reasons (3) to (5) are common for JPEG and JPEG2000, but only JPEG2000 satisfies reasons (1) and (2) for excellent image reproduction which is requested by motion picture people

\subsection{Real-time DECODER}

The DECODER can perform the real-time decompression at a speed of 500M pixels per second, using parallel JPEG2000 processing elements. As a JPEG2000 processor, Analog Devices Inc. ADV202 is selected. The decoder consists of 2 circuit blocks, a PC/LINUX part with GbE interface, and newly developed JPEG2000 decoder boards shown in figure 4. 4 chips of ADV202 are installed in each board. Total 4 boards are installed on the PCI-X-bus in order to process 24 frames of $4 \mathrm{~K} \times 2 \mathrm{~K}$ pixel up to 36 -bit RGB color images $(4: 4: 4)$ in a second. At the same time, This board supports 10 bits $\mathrm{YCbCr}(4: 2: 2)$ mode and transfer function from $\mathrm{YCbCr}$ to RGB is installed. This kind of flexibility is obtained by the FPGA based circuit design.

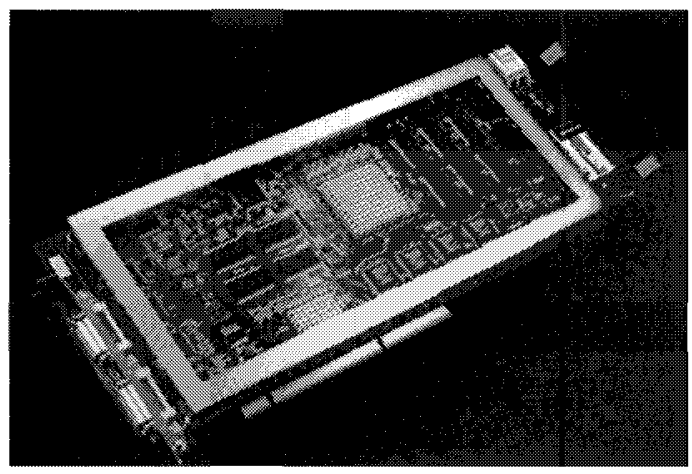

Figure 4. Photograph of JPEG2000 decoder board 
The PC part receives the coded streams of $200 \mathrm{M}$ to $500 \mathrm{Mbps}$, then transfers them to JPEG2000 decoder boards. The PC part consists of dual CPUs running with LINUX. A control program runs as an application that consists of two threads that share the PC's main memory as a large data buffer. One thread reads the data received from GbE-NIC, and the other reformats and forwards them to each of four decoder boards. The buffer size is only limited by the main memory size of the PC, usually set to 200MB.

\subsection{LCD Projector}

The prototype projector itself was developed by JVC using D-ILA technology. The high picture quality of D-ILA is derived from the advanced LCOS (Liquid Crystal on Silicon) technology and a high-precision optical system. The major features of D-ILA are high brightness, high resolution, high contrast ratio, analog gradation and high-speed response. The SHD LCD projector uses three pieces of 3840x2048 pixel reflective D-ILA panels for each RGB 10-bit colors, and its size is 1.7 inches. The effective brightness exceeds 5000 ANSI lumens by using a $1600 \mathrm{~W}$ xenon lamp, which is bright enough to show images on as large as 300inch diagonal screens. The refresh rate of the projector is chosen to $96 \mathrm{~Hz}$. This high refresh rate thoroughly eliminate flicker, and is compatible to 24fps movie. Every frame of decoder output is simply displayed for times in the projector without any interpolation between adjacent frames.

\subsection{PC Video Server}

The PC server consists of LINUX running on dual CPUs (Pentium III, 1.44GHz), an IDE-RAID (200GB x 6, RAID0, striping mode), and a GbE NIC. The movie films are digitized to a large set of still images, and compressed in advance. The original movie data are divided into $960 \times 512$ pixel image tiles, and they have 30 bits RGB color components. A data transfer command reads the data from the RAID and writes them periodically to the GbE NIC. Like the DECODER control program, a large size shared buffer is used in the server in order to enhance the maximum transmission rate by averaging the disk read speed. Contrary to an ordinal streaming system that runs by its own clock, the data rate of the server is precisely controlled by the DECODER via acknowledge signal. This is because the decoder generates the master-clock of the movie system of $24 \mathrm{~Hz}$ to yield smooth replay without the lack or duplicate of frame 


\section{EXPERIMENTAL RESULTS}

The quality of SHD movies and performance of the transmission system are evaluated by using long movie data that are digitized and compressed from a variety of actual movies.

\subsection{Digital Cinema Data Acquitision}

To evaluate the SHD movie system, a lot of movie sequences are required to compare with the conventional film movies, So we provided various types of high quality movie data sequences with less blurs, scratches, nor grain noises. Some of the sequences are digitized from original negative films, such as "Circle of Love" provided by ARRI and short test sequences provided by Hollywood studios. Others are from inter-positive (IP) films, such as "Tomorrow's Memory" shot by NTT, and dupe-negative (DN) films, such as "Tomb Raider" of full Hollywood movie. Usually, we use an IMAGICA's "Imager XE" film scanner to digitize these films. This scanner can yield image files of $4096 \times 3012$ pixel as maximum size with RGB 10 bit $\log$ Cineon format and it takes 10 seconds per frame for scanning. This is a very time consuming process of data acquisition. Recently, Thmoson Grass Valley announced the development of high speed film digitizer. This kind of high speed film digitizer is indispensable to make large variety of digitized cinema contents to form a cinema archiving with reasonable costs in the near future.

Master image file is obtained from this 10 bit log Cineon format image files through the color correction process. As master image file format, we use RGB $30 \mathrm{bit}$ TIFF file for each frame. In the case of "Tomb Raider" of 101 minutes movie, the size of master file is 4.5 Tera Bytes from 144,000 frames.

As far as sound material, we don't apply any compression technologies and we use uncompressed sequence. Usually, master sound can be obtained as the TASCAM DA98 tapes. The 6-channel sound tracks are extracted from this tape. The digital sound data are stored in the Linux/PC server as WAV files and transmitted as IP stream with image data. As the highest sound quality, 24 bit per channel with $96 \mathrm{KHz}$ sampling is available for the play back of orchestra and musical.

\subsection{Network Transmission}

We selected TCP for the connection protocol from server to real-time DECODER. TCP is adequate for the stable connected transmission and best method to share the bandwidth of IP router based network. Within the multiplex cinema, it is very easy to use. But for large RTT (Round trip time) network, it is very difficult to extract its full performance. In order to verify the performance, we 
had an experiment with a long distance high-speed IP network environment called Internet 2, on 29th October, 2002. In this case, we used the second generation of our SHD digital cinema distribution system $[5,6]$.

The network configuration is shown in figure 5 . We set up the server in the Electronic Visualization Laboratory (EVL) at the University of Illinois, Chicago (UIC). The real-time DECODER and the projector were installed at the Robert Zemeckis Center of the School of Cinema-Television at the University of Southern California (USC), Los Angeles. The distance between the server and the decoder was more than $3000 \mathrm{~km}$. There were six router hops between them. The RTT (Round Trip Time) of the network was measured to be $59 \mathrm{~ms}$. The target transmission rate is $300 \mathrm{Mbps}$. Many power users were sharing Internet 2 while our experiments.

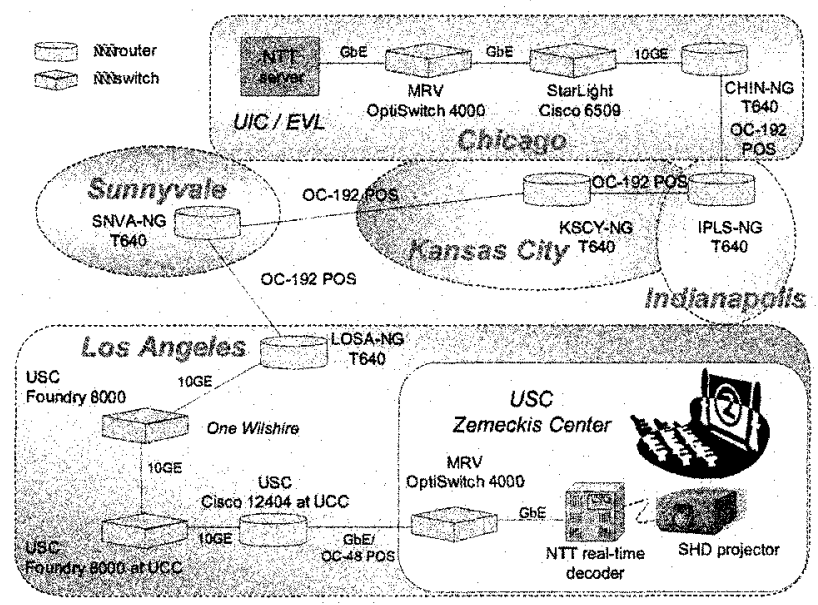

Figure 5. Network configuration of long distance transmission with Internet2

To overcome the long distance, we applied (1) a large TCP window, 4 MByte, (2) multiple TCP connections, and (3) a shaping control function. The TCP window size is the amount of data able to be sent without acknowledgement. There are theoretical limitations to TCP-window-based flow control. The configuration guideline is "window size > Required TCP throughput $x$ RTT ". The TCP window size of the system was extended to 4 MByte from its initial 64 KByte value. Measured throughput was increased from $8 \mathrm{Mbps}$ to $50 \mathrm{Mbps}$. Theoretically, extension of window size is enough, but it is impossible to extract real high speed.

To improve the performance, we increase the number of TCP connections between the server and the real-time CODEC. The server application divided the movie data into equal segments and sequentially wrote them to multiple TCP sockets. As the number of TCP connections increased, it was confirmed that 
throughput went up. Finally, using 64 TCP connections, we could get $200 \mathrm{Mbps}$. But the stream's bit rate could not be raised even when the number of connections was increased more than 64 .

We used an application traffic monitor to observe the traffic pattern to a resolution of $1 \mathrm{~ms}$. We found that the nature of data transmission was very bursty. To suppress the burstiness, a shaping control function for the data transmission was built into the socket writing process of the server application. As a result, transmitted movie data traffic reach $300 \mathrm{Mbps}$. We could succeed in transmitting SHD movie over $3000 \mathrm{Km}$ at $300 \mathrm{Mbps}$ with TCP/IP protocol. To implement adequate functions, TCP can be applied to long distance transmission.

\section{CONCLUSIONS}

We have developed an SHD digital cinema system that offers the resolution of $3840 \times 2048$ pixels and the quality of RGB 30 bits. We have exerted concerted efforts in realizing a complete digital cinema system that fully match the quality of $35 \mathrm{~mm}$ film. Now the technology of optical network has matured and the new era of broadband network is coming. We believe that a lot of image service will appear using this kind of high quality digital content distribution platform.

\section{REFERENCES}

[1] S. Ono et al, N. Ohta, and T. Aoyama, "All-digital super high definition images", Signal Processing: Image Communication 4, pp. 429-444 1992.

[2] S. Ono and J. Suzuki, "Perspective for Super-High-Definition Image Systems," IEEE Communication Magazine, pp. 114-118, Jun. 1996

[3] ISO/IEC JTC1/SC29/WG1 15444-1:2000 "JPEG 2000 image coding system -- Part 1", 2000

[4] T. Fujii, M. Nomura, et al. "Super High Definition Digital Movie System", SPIE VCIP'99, Vol. 3653, pp. 1412-1419, Jan. 1999

[5] T. Fujii et al. "IP Transmission System for Digital Cinema Using 2048 scanning line resolution", IEEE Globecom 2002, GEN-01-2, Nov. 2002

[6] T. Fujii, et al. "Super High Definition Digital Cinema Delivery System with 8 Million Pixel Resolution", RICHMEDIA 2003, pp.79-88, Lausanne, Switzerland, October 2003 